\title{
NEFROPATÍA DIABÉTICA COMO FACTOR DE RIESGO ASOCIADO A INFECCIÓN DEL TRACTO URINARIO, SERVICIO DE MEDICINA DEL HOSPITAL ULDARICO ROCCA FERNÁNDEZ, 2011 - 2015
}

\author{
DIABETIC NEPHROPATHY AS RISK FACTOR ASSOCIATED WITH URINARY TRACT INFECTION, MEDICAL \\ SERVICE OF THE HOSPITAL ULDARICO ROCCA FERNANDEZ, 2011 - 2015
}

Maggie Martinez-Palomino,a, Edwin Castillo-Velarde 2,b

\begin{abstract}
RESUMEN
Introducción: La diabetes mellitus es una de las patologías más prevalentes, afectando hasta el 2014 a un 9\% de la población mundial y en el Perú el $4.3 \%$ de la población adulta ya es diabética. Estos pacientes son más susceptibles a enfermedades infecciosas, siendo la del tracto urinario la más frecuente, considerándose complicada, lo que significa que deben recibir manejo hospitalario, conllevando a disminución de la calidad de vida de los mismos. Objetivo: Determinar si la nefropatía diabética es un factor de riesgo para la prevalencia de infección del tracto urinario en pacientes hospitalizados en el servicio de medicina del Hospital Uldarico Rocca Fernández. Métodos: Es un estudio observacional, analítico de corte longitudinal, tipo caso y control; la información se recolectó mediante una ficha de datos utilizando las historias clínicas de los pacientes hospitalizados en el servicio de medicina del Hospital Uldarico Rocca Fernández en el periodo 2011-2015. La muestra se obtuvo por muestreo probabilístico, siendo el tamaño de esta de 360, con una proporción de casos y controles 1:1. Resultados: La nefropatía diabética obtuvo un OR=8.62 (IC95\% 4.78 - 15.57), la macroalbuminuria obtuvo un $\mathrm{OR}=5.75$ (IC95\% 2.99 - 11.06), el pH alcalino obtuvo un OR=5.42 (IC95\% 3.22 - 9.13) y el control glucémico obtuvo un OR=5.44 (IC95\% 3.44 - 8.88). Conclusión: La nefropatía diabética se asocia a mayor prevalencia de ITU en pacientes hospitalizados en el servicio de medicina del Hospital Uldarico Rocca Fernández.
\end{abstract}

Palabras clave: Infección del tracto urinario; Nefropatía diabética; pH urinario; Grados de proteinuria; Control glucémico; Pacientes diabéticos. (fuente: DeCS BIREME)

\begin{abstract}
Introduction: Diabetes mellitus is one of the most prevalent pathologies, affecting up to 2014 to $9 \%$ of the world population and in Peru, 4,3\% of the adult population is already diabetic. These patients are more susceptible to infectious diseases, urinary tract infection are the most complicated frequently involve, these patients receive hospital treatment, decreasing their life quality. Objective: To determine if diabetic nephropathy is a risk factor for the prevalence of urinary tract infection in hospitalized patients at the Uldarico Rocca Fernandez Hospital. Methods: It is an observational, analytical study of longitudinal cut, case and control type; the information was collected through a data sheet using the medical records of patients hospitalized in the medical service of the Hospital Uldarico Rocca Fernandez in the period 2011-2015. The sample was obtained by probabilistic sampling, sample size of 360 , with a proportion of cases and controls 1:1. Results: Diabetic nephropathy had an OR $=8.62$ (95\% Cl 4.78 - 15.57), macroalbuminuria obtained an $\mathrm{OR}=5.75(95 \% \mathrm{Cl} 2.99-11.06)$, the alkaline pH had an OR= 5.42 (95\% Cl 3.22-9.13) and the Glycemic control obtained an OR $=5.44$ ( $95 \% \mathrm{Cl} 3.44$ - 8.88). Conclusion: Diabetic nephropathy is associated with a higher prevalence of UTI in patients hospitalized in the medical service of the Hospital Uldarico Rocca Fernandez.
\end{abstract}

Key words: Urinary tract infection; Diabetic nephropathy; Urinary pH; Degrees of proteinuria; Glycemic control; Diabetic patients. (source: MeSH NLM)

\footnotetext{
${ }^{1}$ Hospital Uldarico Rocca Fernández, Lima-Perú.

a Interno de Medicina.

${ }^{2}$ Hospital Nacional Guillermo Almenara Irigoyen, Lima-Perú.

b Especialista en Nefrología y Transplante Renal.
}

Citar como: Maggie Martinez-Palomino, Edwin Castillo-Velarde. Nefropatía diabética como factor de riesgo asociado a infección del tracto urinario, servicio de medicina del Hospital Uldarico Rocca Fernández, 2011 - 2015.[Artículo Original].2019;19(1):40-45. (Enero 2019). DOI 10.25176/RFMH.v19.n1.1791

( ) Los autores. Este artículo es publicado por la Revista de la Facultad de Medicina Humana, Universidad Ricardo Palma. Este es un artículo de Open Access distribuido bajo los términos de la Licencia Creative Commons Atribución-NoComercial-Compartirlgual 4.0 Internacional.(http://creativecommons.org/licenses/by-nc-sa/4.0/), que permite el uso no comercial, distribución y reproducción en cualquier medio, siempre que la obra original sea debidamente citadas. Para uso comercial, por favor póngase en contacto con revista.medicina@urp.pe 


\section{INTRODUCCIÓN}

La diabetes mellitus es una de las patologías más prevalentes, afectando hasta el 2014 a un $9 \%$ de la población mundial' y se estima que en el Perú el $4.3 \%$ de la población adulta ya es diabética². En la actualidad, la diabetes causa estragos en varios órganos, siendo entre ellos, el riñón uno de los principales órganos afectados, produciéndose nefropatía diabética que es la principal causa de enfermedad renal crónica terminal, por ende, es la primera causa de muerte en los pacientes diabéticos², estimándose que para el 2030 será la séptima causa de muerte en el mundo'.

Es de considerar que aquellas personas que presentan el diagnóstico de diabetes son más susceptibles a enfermedades infecciosas, siendo las del tracto urinario las que se presentaron con mayor frecuencia, se podría explicar porque dichos pacientes se encuentran en un estado de inmunodepresión ${ }^{3}$, pero a la vez existen muchos otros factores que colaboran para la presentación de dichas infecciones, siendo uno de estos, la nefropatía diabética que en la práctica clínica se ve relacionada con mayores tasas de infecciones en el tracto urinario ${ }^{4}$.

La infección del tracto urinario en pacientes diabéticos se considera complicada, lo que significa que debe recibir manejo hospitalario, conlleva a disminución de la calidad de vida del paciente, mayor gasto económico para el manejo, conduce a un mal control de glicemias, por ende mayor daño en órganos blancos ${ }^{3,5}$, lo que daría como resultado un círculo vicioso.

Los factores que se tomaron en cuenta son proteinuria, el $\mathrm{pH}$ urinario y el mal control glucémico que son parámetros considerados dentro de la nefropatía diabética, y al evaluarse individualmente podrá establecerse cuál de los tres es el más asociado a prevalencia de infección urinaria en los pacientes diabéticos y según ello, establecer qué medidas se pueden tomar para poder modificarlos y de esta manera disminuir el riesgo.

En el presente trabajo, se aportó tanto al ámbito médico como al ámbito social datos importantes sobre dicha asociación, ya que al existir pocos estudios que desarrollen este tema, se busca incentivar la investigación de aquellos factores que podrían estar asociados al deterioro de la salud de aquellos pacientes crónicos, para darlos a conocer; que se haga un mejor seguimiento a estos pacientes para reducir los riesgos, y de esta manera evitar el deterioro de la calidad de vida de los mismos. Se buscó también promover en el sector salud del Perú la medicina preventiva que en los últimos años ha tomado gran importancia en el mundo.

Por lo mencionado, en la presente investigación buscamos ddeterminar si la nefropatía diabética es un factor de riesgo para la prevalencia de infección del tracto urinario en pacientes hospitalizados en el servicio de medicina del Hospital Uldarico Rocca Fernández en el período 2011-2015.

\section{MÉTODOS}

Se realizó un estudio de tipo observacional, analítico de corte longitudinal, de tipo casos y controles. La muestra de estudio estuvo conformada por 320 pacientes diabéticos de ambos sexos con edad promedio de 66 años, hospitalizados en el servicio de medicina del hospital Uldarico Rocca Fernández durante el periodo 2011-2015, que se dividieron en dos grupos: casos y controles en relación 1:1 pareados por edad y sexo.

\section{Criterios de inclusión:}

Casos: Pacientes diabéticos que presentaron infección del tracto urinario (ITU).

Controles: Pacientes diabéticos que no tenían diagnóstico de ITU.

\section{Criterios de exclusión:}

Pacientes con diagnóstico previo de hipertensión arterial, urolitiasis y/o vejiga neurogénica.

La información fue proveniente de sus historias clínicas mediante una ficha de datos sencilla donde se tuvo en cuenta la cantidad de proteinuria, $\mathrm{pH}$ en orina y los valores de control glucémico. El análisis de los datos estadísticos se realizó utilizando los programas IBM SPSS Statitics versión 22 y Microsoft Excel.

\section{RESULTADOS}

Se registró un total de 320 pacientes, hospitalizados en el servicio de medicina del hospital Uldarico Rocca Fernández durante el periodo 2011-2015, la distribución de la muestra según el grupo casos y controles se describe en la tabla 1, totalizando 160 pacientes en cada grupo en una relación 1:1, donde el $52.5 \%$ son del sexo femenino y $47.5 \%$ pertenecen al sexo masculino.

Tabla 1. Características generales del grupo casos y controles.

\begin{tabular}{lcc}
\multicolumn{1}{c}{ VARIABLES } & CASOS & CONTROLES \\
\hline Número & 160 & 160 \\
Sexo femenino & $84(52.5 \%)$ & $84(52.5 \%)$ \\
Sexo masculino & $76(47.5 \%)$ & $76(47.5 \%)$ \\
Edad (años) & 63 & 63 \\
ITU positivo & 160 & 0 \\
\hline
\end{tabular}


La prevalencia de infección del tracto urinario en los pacientes diabéticos que pertenecían al grupo de estudio es de $22 \%$ frente a un $78 \%$ que acudía por otras patologías, lo que se evidencia gráficamente en la figura 1.

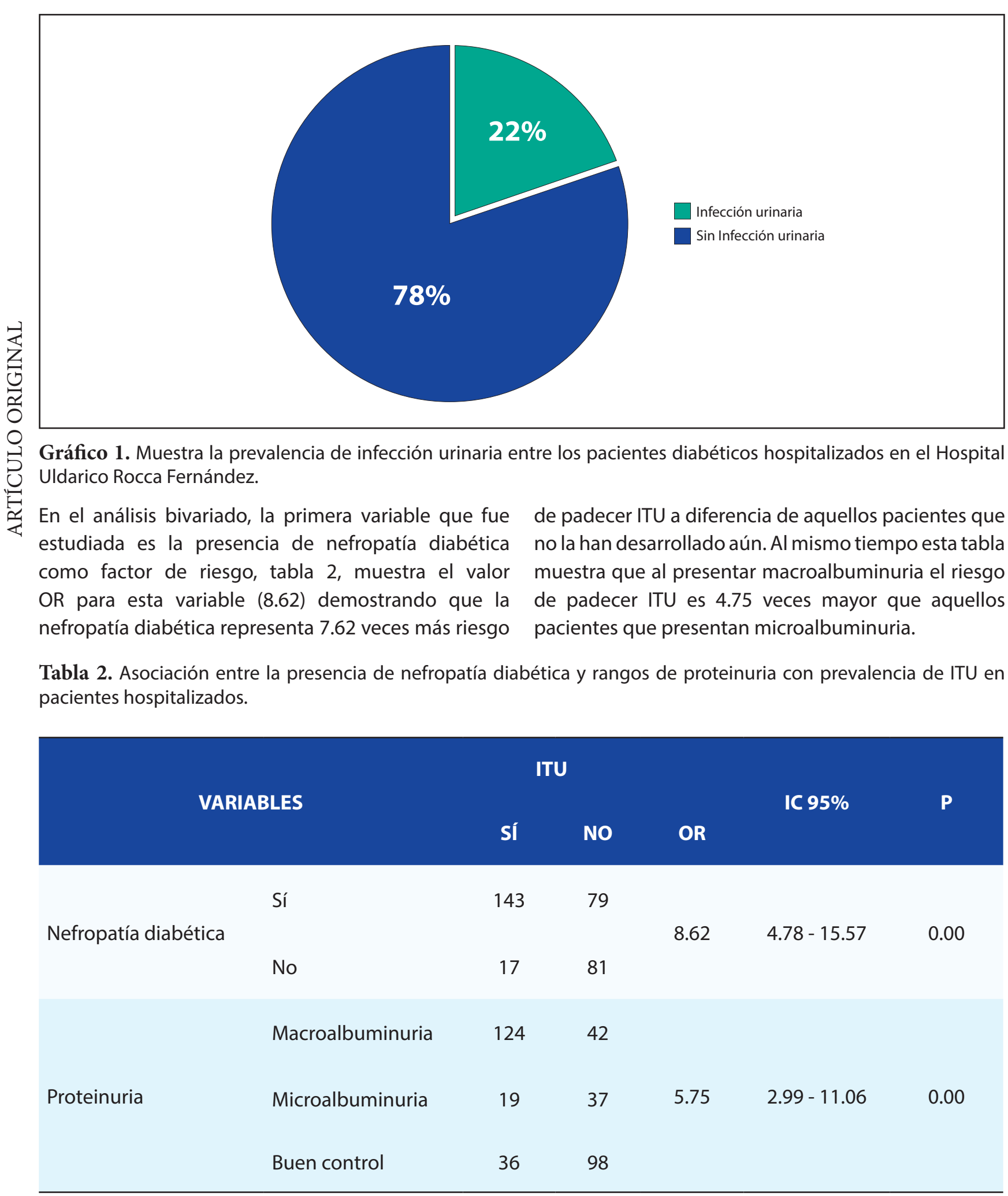

Las otras dos variables que fueron estudiadas son el $\mathrm{pH}$ urinario y el control de las glicemias, observamos en la tabla 3, que al presentar $\mathrm{pH}$ alcalino en la orina el riesgo de presentar ITU aumenta en 4.42 veces a diferencia de los pacientes que conservan $\mathrm{pH}$ ácido en la orina. A la vez se mostró que un mal control de la glicemia representa 4.44 veces más riesgo de presentar ITU en aquellos pacientes diabéticos. 
Tabla 3. Asociación entre pH urinario y control glucémico con prevalencia de ITU en pacientes hospitalizados.

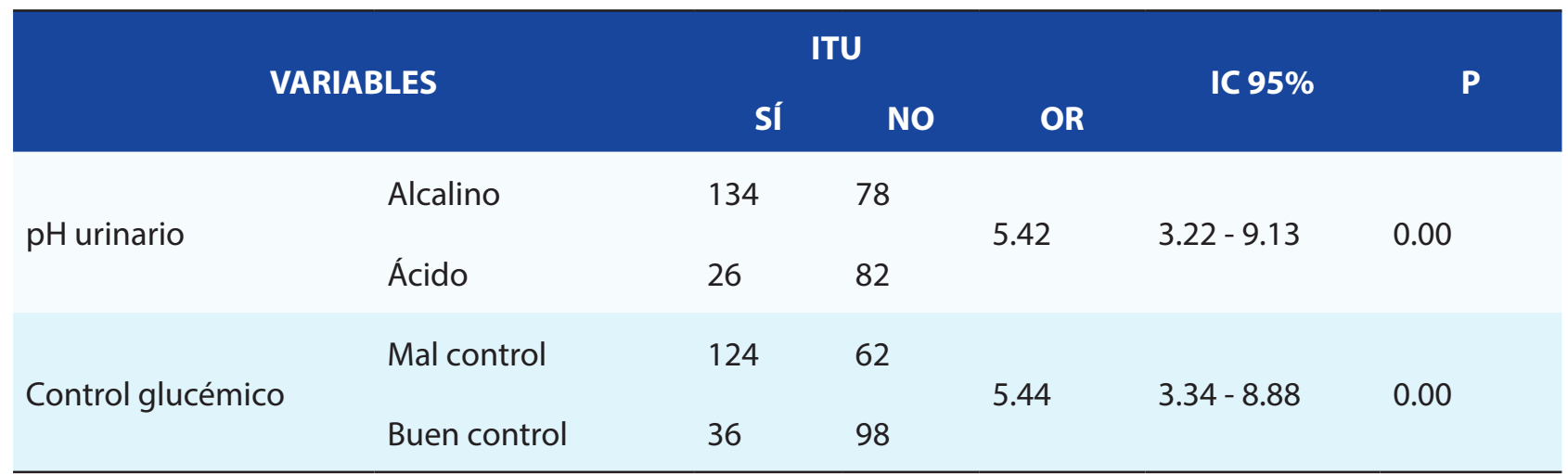

Por último, en el análisis multivariado, que se muestra diabéticos es el mal control glicémico, con un OR=5.58, en la tabla 4, determinó que aquel factor que tiene significativamente estadístico (valor $\mathrm{p}=0.00$ ).

más relación con la presentación de ITU en paciente

Tabla 4. Análisis de regresión logística entre los factores de riesgo y la presencia de infección del tracto urinario en pacientes diabéticos hospitalizados.

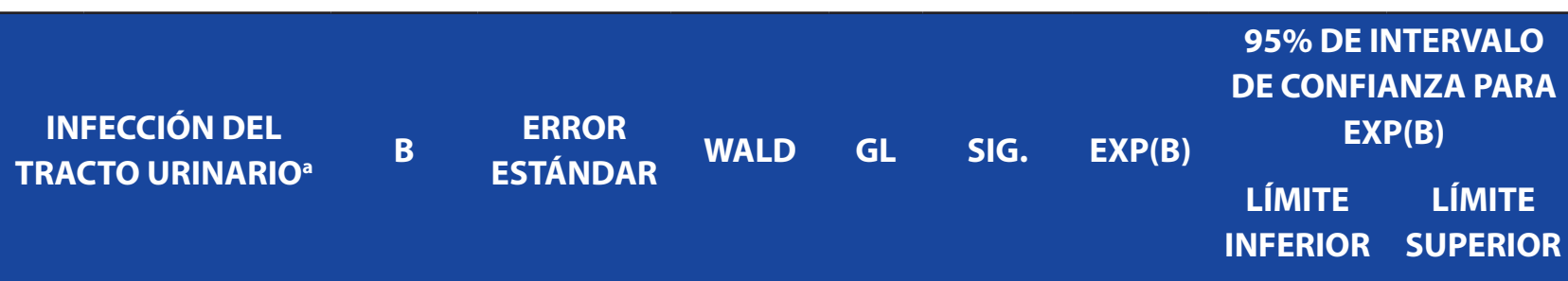

$\begin{array}{lcccccccc}\text { Interceptación } & -2,722 & 0,373 & 53,220 & 1 & 0 & - & - & - \\ \text { [Nefro=1] } & 1,297 & 0,451 & 8,281 & 1 & 0,004 & 3,66 & 1,512 & 8,855\end{array}$

$[$ Nefro $=2] \quad 0^{b}$

$[$ Prot $=0]$

$0^{\mathrm{b}}$

$[$ Prot $=1]$

1,3

0,394

10,887

0,001

3,671

1,696

7,948

Sí

[Prot $=2]$

$0^{b}$

$[\mathrm{pH}=1]$

0,154

0,410

0,142

0,707

1,167

0,522

2,606

$[\mathrm{pH}=2]$

[ControlGlu=2]

1,719

0,294

34,175

1

0

5,58

3,136

9,93 


\section{DISCUSIÓN}

El presente estudio, y en relación a algunos estudios anteriores, se buscó determinar el grado de asociación entre la nefropatía diabética y la presencia de infección del tracto urinario, se observó que la nefropatía diabética tiene un riesgo de 7.62 veces más de presentar ITU a diferencia de aquellos pacientes que no desarrollaron nefropatía diabética $(\mathrm{OR}=8.62$, IC95\% $=4.78$ - 15.57).

Resultados similares reportaron Oma Nitzan, Mazen Elias, Viviana Chazan y colaboradores, en su estudio "Urinary tract infections in patients with type 2 diabetes mellitus: review of prevalence, diagnosis, and management" ${ }^{\prime \prime}$, encontraron un $\mathrm{RR}=1.42$ para esta variable. Si bien no son las mismas medidas de asociación, ambos resultados son congruentes, indicando que el desarrollar nefropatía diabética representó un mayor riesgo para ITU, probablemente se deba a que el daño renal produce proteinuria, $\mathrm{pH}$ alcalino y es un indicador indirecto de mal control glicémico. Al analizar el IC95\% se obtiene que este resultado fue estadísticamente significativo.

En relación al rango de proteinuria se observó que la macroalbuminuria tuvo 4.75 veces más riesgo de haber presentado ITU en comparación con los que solo presentaron microalbuminuria (OR $=5.75$, IC95\% $=2.99-11.06)$. No se han encontrado trabajos de investigación que hayan estudiado dicha relación exactamente pero Al-Rubeaan KA, Moharram O, Al-Naqeb D, Hassan A, Rafiullah MR en su estudio "Prevalence of urinary tract infection and risk factors among Saudi patients with diabetes ${ }^{\prime \prime 10}$ encontraron que la microalbuminuria tuvo un $\mathrm{RR}=1.4$ en contraste a pacientes sin proteinuria, demostró que la aparición de proteínas en orina significó un mayor riesgo para ITU y al aumentar la cantidad de proteínas en orina el riesgo es mayor. Al analizar el IC95\% y el valor $p$ se obtiene que este resultado fue estadísticamente significativo.

Además, referente al pH urinario, se determinó que el $\mathrm{pH}$ alcalino representó 4.42 veces más riesgo de padecer ITU en pacientes diabéticos a diferencia de aquellos que tuvieron un $\mathrm{pH}$ urinario ácido, habiendo sido el resultado estadísticamente significativo $(\mathrm{OR}=$ $5.42, \mathrm{IC} 95 \%=3.22-9.13, \mathrm{p}<0.05)$. A pesar de haberse comprobado esta asociación, no se contó con estudios que al compararse avalaran o discordaran con el presente estudio, quedando abierta la posibilidad que se realicen más estudios referentes al tema, si bien ante el análisis bivariado esta variable mostró una asociación estadísticamente significativa, sin embargo en el análisis multivariado, se obtuvo que la asociación no fue estadísticamente significativa ya que el valor de p fue 0.7 .

La última variable a evaluar fue el control glucémico, dando como resultado que el mal control de la glicemia tuvo 4.44 veces más riesgo para ITU en comparación con aquellos pacientes que llevaron un buen control, se observó que este resultado fue estadísticamente significativo $(O R=5.44, \mathrm{IC} 95 \%=3.34-8.88, \mathrm{p}<0.05)$. Así mismo Suzanne Geerling, Vivian Fonseca, David Castro-Diaz, James List y Shamik Parikh en su estudio "Genital and urinary tract infections in diabetes: Impact of pharmacologically-induced glucosuria" encontraron que un pobre control glucémico fue un factor de riesgo para presentar infección en el tracto urinario. Esto concuerda con el resultado del presente estudio, ya que a peor control de las glicemias es mayor el compromiso de órganos blancos.

\section{CONCLUSIÓN}

En base a los resultados obtenidos concluimos que la nefropatía diabética sí es un factor de riesgo para la presentación de infección del tracto urinario en aquellos pacientes; además se encontró que los demás factores: rangos de proteinuria, $\mathrm{pH}$ urinario y control glucémico juegan un rol importante, predominando el mal control glicémico, que represento el mayor factor de riesgo.

Contribuciones de autoría: La autora participó en la generación, redacción y aprobación final del artículo original, además en la recolección de datos.

Financiamiento: Autofinanciado.

Conflicto de interés: La autora declara no tener conflictos de interés con la publicación de este artículo.

Recibido: 23 de Agosto del 2018

Aprobado: 15 de diciembre del 2018

Correspondencia: Maggie Fiorella Martinez Palomino

Dirección: Av. Honorio Delgado 555-Urb. Ing. San Martin de Porres, Lima-Perú Teléfono: +51973874297

Correo:maggie23_9@hotmail.com 


\section{REFERENCIAS BIBLIOGRÁFICAS}

1. Organización Mundial de la Salud. Diabetes [monografías en internet] ${ }^{*}$ secretaria general de la OMS; 2015 [02/07/16] $\otimes$ disponible en: http:// www.who.int/mediacentre/factsheets/fs312/es/

2. Ministerio de Salud. Día mundial de la Diabetes [monografías en internet] $\otimes$ portal web MINSA; 2014 [04/07/16]* disponible en: http:// www.minsa.gob.pe/portada/Especiales/2014/diabetes/

3. Fauci A., Kasper D., Longo D., Braunwald E., Hauser S., Jameson J., et al. Harrison. Principios de medicina interna.17a ed. México: Mc Graw Hill; 2009.

4. Orna N., Mazen E., Vibiana C., Walid S. Urinary tract infections in patients with type 2 diabetes mellitus: review of prevalence, diagnosis, and management. Diabetes, Metabolic Syndrome and Obesity: Targets and Therapy 2015; 8: 129-136.

5. Greerlings S., Fonseca V., Castro-Díaz D., James L., Parikh S. Genital and urinary tract infections in diabetes: Impact of pharmacologically-induced glucosuria. Diabetes research and clinical practice 2014; 103: 373-381.

6. Pesantez Mendez C., Ruilova Blacio J. Prevalencia de infección de vías urinarias en pacientes con diagnóstico de diabetes mellitus tipo 2 . Ingresados en el departamento de medicina interna del hospital Vicente Corral Moscoso durante el año 2011 y factores asociados. [Tesis previa a la obtención del título médico]. Cuenca: Universidad de Cuenca, Facultad de ciencias médicas, Escuela de medicina; 2013.

7.Villarroel P., Parra X., Ardiles L. Prevalencia y clasificación de enfermedad renal crónica en pacientes con diabetes mellitus tipo 2 en el centro comunitario de salud familiar Pantanosa, Frutillar. Rev. Med. Chile 2012; 140: 287-294.

8. Herrera-Añazco P., Hernández A., Mezones-Holguin E. Diabetes mellitus y nefropatía diabética en el Perú. Nefrologia, Diálisis y Trasplante 2015; 35 (4): $229-237$.

9. Torres A., Zacarías R. Nefropatía diabética. Rev Hosp Gral Dr. M Gea González 2002; 5 (1-2): 24-32.
10. Al-Rubeaan K., Moharram O., Al-Naqeb D., Hassan A., Rafiullah M. Prevalence of urinary tract infection and risk factors among Saudi patients with diabetes. Worl J Urol 2013; 31(3): 573-578.

11. Barrot J., Gonzáles A. Diabetes mellitus tipo 2. AMF 2012; Jun: 1-2.

12. Borregales L., Giordano F., Contreras L. Primer consenso venezolano de infección urinaria. $1^{\text {a }}$ ed. Caracas: Sociedad Venezolana de Urología; 2011.

13. Chávez $\mathrm{O}$. Uso de tiras reactivas para el cribado de Nefropatía en mayores de 60 años. Rev Cient Cienc Med 2013; 16(2): 26-30.

14. Feki M., Kamoun M., Hadj F., Bouaziz Z., Charfi N., Mnif F., et al. Complicated urinary tract infections associated with diabetes mellitus: Pathogenesis, diagnosis and management. Indian Journal of Endocrinology and Metabolism 2013; 17(3): 442-445.

15. Fünfstück R., Nicolle L., Hanefeld M., Naber K. Urinary tract infection in patients with diabetes mellitus. Clinical Nephrology 2012; 77(1): 40-48.

16. González A., Dávila R., Acevedo O., Ramírez M., Gilbaja S., Valencia C., et al. Infección de las vías urinarias: prevalencia, sensibilidad antimicrobiana y factores de riesgo asociados en pacientes con diabetes mellitus tipo 2 . Revista Cubana de Endocrinología 2014; 25(2): 57-65.

17. Gonzales N., Rodríguez E., Manrique H. Características clínicas y factores asociados a morbilidad intrahospitalaria en los pacientes con diabetes mellitus tipo 2. Rev Soc Peru Med Interna 2013; 26 (4): 159-165.

18. Ramos W., López T., Revilla L., More L., Huamaní M., Pozo M. Resultados de la vigilancia epidemiológica de diabetes mellitus en hospitales notificantes del Perú, 2012. Rev Peru Med Exp Salud Pública 2014; 31(1): 9-15.

19. Palacios Villegas E., Ruilova Blacio J. Diabeticos tipo 2 en complicación aguda con infecciones adquiridas en la comunidad ciudad hospitalaria "Dr. Enrique Tejera" septiembre 2014 - mayo 2015. [Tesis previa a la obtención del título de especialista en medicina interna]. Valencia: Universidad de Carabobo, Facultad de ciencias de la salud, Dirección de postgrado; 2015.

\section{Indizado en: latindex}

http://www.latindex.org/latindex/ficha?folio=14280<smiles>C1CC2CCC1C2</smiles>

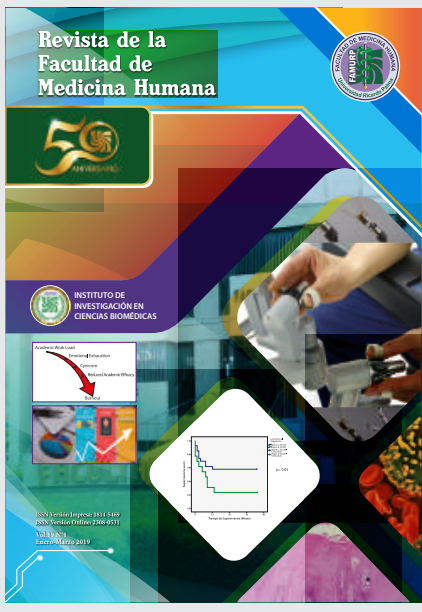

